\title{
Effect of Fat and CHO Meals on Intermittent Exercise in Soccer Players
}

Authors

Affiliations

\section{A. T. Hulton ', J. P. Edwards ${ }^{2}$, W. Gregson ${ }^{1}$, D. MacLaren ${ }^{1}$, D. A. Doran}

${ }^{1}$ Research Institute for Sport \& Exercise Sciences, Liverpool John Moores University, Liverpool, United Kingdom ${ }^{2}$ School of Sport, Health and Exercise Sciences, Bangor University, Bangor, United Kingdom
Key words

high fat meals

pre-exercise meals

intermittent exercise

association football

accepted after revision

June 16, 2012

\section{Bibliography}

DOI http://dx.doi.org/

$10.1055 / \mathrm{s}-0032-1321798$

Published online:

September 12, 2012

Int J Sports Med 2013; 34:

165-169 @ Georg Thieme

Verlag KG Stuttgart · New York

ISSN 0172-4622

\section{Correspondence}

\section{Andrew Thomas Hulton}

Sport \& Exercise Sciences Liverpool John Moores University

Tom Reilly Building

L3 3AF Liverpool

United Kingdom

Tel.: +44/151/231 4358

Fax: $+44 / 151 / 2314353$

a.t.hulton@ljmu.ac.uk

\section{Abstract}

$\nabla$

Pre-exercise meals containing carbohydrates (CHO) are recommended to athletes, although there is evidence to suggest that a high fat meal prior to exercise increases utilisation of fats yet may not adversely affect performance. This study investigated the effect of a high fat and high $\mathrm{CHO}$ pre-exercise meal prior to high intensity intermittent exercise. Ten male recreational soccer players performed a soccer specific protocol followed by a $1 \mathrm{~km}$ time trial $3 \frac{1}{2} \mathrm{~h}$ after ingesting one of 2 test meals, high fat meal (HFM) or a high $\mathrm{CHO}$ meal (HCM). Blood glucose, fatty acids (FA), glycerol, $\beta$-hydroxybutyrate, lactate and insulin were assessed prior to the meal, pre-exercise, half-

\section{Introduction}

\section{$\nabla$}

The need to maintain glycogen stores during soccer has been previously illustrated [1] as performance can be severely affected as muscle glycogen levels are depleted. Similarly previous research [25] found a significant reduction in muscle glycogen after 90 min of match play, and observed that distance covered was reduced for players expressing lower levels of muscle glycogen compared to those with higher levels. More recently, authors [15] investigated muscle glycogen levels pre and post match, and observed a decrease $\left(449 \pm 23-255 \pm 22 \mathrm{mmol} \cdot \mathrm{kg}^{-1} \mathrm{~d}\right.$. w. $)$ in overall muscle glycogen levels and found that $47 \%$ of the muscle fibres were completely or almost empty of glycogen after the match. It is clear that muscle glycogen levels are reduced playing soccer, and that this may impact on the high intensity work during the game, especially the latter stages of matches.

In order to optimise muscle glycogen levels, the provision of diets with high carbohydrate $(\mathrm{CHO})$ content is advocated. It has been observed [3] time, and post-exercise, whilst rates of $\mathrm{CHO}$ and fat oxidation were determined at 4 time points during the exercise as well as heart rate (HR) and rating of perceived exertion (RPE). Significant increases in FA, glycerol, $\beta$-hydroxybutyrate and fat oxidation after the HFM were observed, while $\mathrm{CHO}$ oxidation was significantly higher following the HCM $(\mathrm{P}<0.05)$. No performance effect was found for the $1 \mathrm{~km}$ time trial (HFM: $228.6+14.4 \mathrm{~s}$; HCM: 229.4+26.5s) (mean+SD). These findings suggest that the type of meal ingested prior to soccer simulated exercise has an impact on metabolism, but not on the subsequent performance as determined in the present study.

that greater running distances during an intermittent running test to exhaustion were found following a $48 \mathrm{~h}$ high $\mathrm{CHO}$ diet relative to a normal diet. Similarly [2] a high $\mathrm{CHO}$ diet prior to small sided soccer games resulted in the players completing a significantly greater amount of high intensity activity. In addition to maintaining a diet high in carbohydrate, several studies have argued that pre-exercise meals favourably impact upon endurance performance [20,26]. Moreover, prolonged intermittent exercise performance may also benefit from the ingestion of pre-performance meals high in CHO. Researchers $[16,17]$ have prescribed a single pre-exercise meal prior to a soccer specific intermittent exercise protocol and found that both high and low glycaemic index $\mathrm{CHO}$ meals improved repeated sprint performance compared to a fasting state.

Following a high CHO meal (HCM) Chryssanthopoulos et al. [7] has suggested a potential 10\% increase in muscle glycogen. Relatively few studies have investigated the effect of a single high fat meal (HFM) compared to a single HCM $[21,22,28]$. Whilst, their findings have indicated 


\begin{tabular}{|lccccc|}
\hline $\begin{array}{l}\text { Food } \\
\text { High Fat }\end{array}$ & Amount & Carbohydrate $(\mathbf{g})$ & Protein $(\mathbf{g})$ & Fat $(\mathbf{g})$ & Energy (kcals) \\
\hline milkshake & & & & & \\
\hline double cream & $200 \mathrm{ml}$ & 22.0 & 8.4 & 7.0 & 179.1 \\
\hline egg fried rice & $50 \mathrm{ml}$ & 1.2 & 1.1 & 37.9 & 349.9 \\
\hline chicken breast & $75 \mathrm{~g}$ & 11.4 & 1.6 & 1.7 & 64.9 \\
\hline korma sauce & $75 \mathrm{~g}$ & 0.3 & 19.6 & 4.4 & 118.3 \\
\hline total & $200 \mathrm{~g}$ & 24.6 & 4.6 & 19.2 & 283.5 \\
\hline High CHO & - & 59.4 & 35.3 & 70.2 & 995.6 \\
\hline apple juice & & & & \\
\hline basmati rice & $590 \mathrm{ml}$ & 65.5 & 0.6 & 0 & 248.0 \\
\hline chicken breast & $100 \mathrm{~g}$ & 71.1 & 9.7 & 1.3 & 317.1 \\
\hline tomato based sauce & $100 \mathrm{~g}$ & 0.3 & 26.1 & 5.8 & 157.7 \\
\hline total & $300 \mathrm{~g}$ & 23.1 & 5.1 & 17.1 & 260.9 \\
\hline
\end{tabular}

Table 1 Meal details for the HFM and HCM.

no improvements in performance for a cycling capacity test [21,22] or for time trial performance [28], critically these studies demonstrate no performance decrements. Underlying these observations is the principle that a HFM enables an increase in fatty acids (FA) availability during the postprandial period and onset of exercise, an increase in the rate of fat oxidation resulting in a matched or enhanced performance.

The purpose of the present study was to investigate the metabolic and performance effects following consumption of a HFM and HCM prior to high intensity intermittent exercise. This approach to nutritional preparation prior to a simulated soccer protocol has not been investigated before, which adds to the novelty of the current study. Both meal design and timing of consumption were intended to convey a realistic scenario commonly observed in professional soccer. We hypothesised that the HFM would promote fat availability and oxidation, and maintain $1 \mathrm{~km}$ time trial performance after $90 \mathrm{~min}$ of high intensity intermittent exercise.

\section{Method \\ $\nabla$}

\section{Participants}

Ten male recreational soccer players all of whom trained twice a week and played a weekly competitive match were recruited for the study (Age: $24.3 \pm 2.3$ yrs [range: $21-26 \mathrm{yrs}$ ]; Body height: $176 \pm 6 \mathrm{~cm}$ [range: $1.60-1.85 \mathrm{~cm}$ ]; Body mass: $74.2 \pm 9.8 \mathrm{~kg}$ [range: $57.95-85.75 \mathrm{~kg}]$ ). Participants provided written informed consent and the study was approved by the Liverpool John Moores University Ethics Committee. Furthermore, the study was performed in accordance with the ethical standards of the International Journal of Sports Medicine [13].

\section{Experimental design}

Each participant was required to attend the laboratory a minimum of 4 occasions (environmental laboratory conditions did not differ between trials, with average temperature and humidity set at $19.7^{\circ} \mathrm{C}$ and $28.9 \%$ ). The first 2 were for familiarisation of the intermittent treadmill protocol, in which participants completed $15 \mathrm{~min}$ of the intermittent treadmill protocol, and then undertook a $1 \mathrm{~km}$ time trial. If the completion time for the time trial was greater than $2 \%$, additional laboratory visits were replicated in the same fashion as described above, until testretest times were within $2 \%$. Only 1 of the participants had to attend the laboratory for a third visit due to his test retest results being $>2 \%$. The final 2 visits were to complete the experimental condition, which were conducted in a counterbalanced manner separated by 7 days.

\section{Test meals}

Test meals ( $\odot$ Table 1$)$ varied in macronutrient content although the total energy intake was similar (HFM - 995 kcal: HCM - 984kcal). The macronutrient content for the HFM was $\mathrm{CHO}: 22 \%$, protein: 14\%, fat: $64 \%$, and for the HCM (Estimation of Glycaemic Index $=41$ [9]) the macronutrient content was $\mathrm{CHO}: 61 \%$, protein: $17 \%$, fat: $22 \%$. Replicating common practise within soccer, participants consumed the same standardised breakfast of cereal and fruit juice (total energy $=377 \mathrm{kcal}$; $\mathrm{CHO}=79 \mathrm{~g}$; Fat $=3 \mathrm{~g}$; Protein $=13 \mathrm{~g}$ ) on both test days at 8 a.m., with participants also abstaining from alcohol and any strenuous exercise for $48 \mathrm{~h}$ prior to the test. The diet $24 \mathrm{~h}$ prior to the test was also kept constant for both trials. Participants consumed on average $346.8 \mathrm{~g} \mathrm{CHO}$ with a total calorie intake of $2303.6 \mathrm{kcal}$, which represents $60 \%$ energy coming from $\mathrm{CHO}$.

\section{Protocol}

Following collection of the first venous blood sample $(20 \mathrm{~mL})$ at approximately 11.10 a.m., participants consumed the test meal at 11.30 a.m. (i.e. $3 \frac{1}{2} 2$ hours before the start of exercise and replicates the current practise of professional soccer players for a 15.00 p.m. kick off). Blood samples were collected from finger prick samples pre-meal, and at 5, 30, 60, 90 and 120 min post meal for the determination of glucose (Glucose Hemocue 201+, Hemocue, Ängelholm, Sweden). During the postprandial period, participants drank $1 \mathrm{~L}$ of water and rested until they returned to the laboratory at 14.30 p.m. for the pre-exercise blood sample.

A standardised warm up consisting of light jogging and static stretching was instigated prior to the start of the protocol at 15.00 p.m. The soccer specific protocol [8] was completed on a motorised treadmill (H/P Cosmos Pulsar, Nussdorf-Traunstein, Germany). This protocol has been used in a previous study investigating pre-exercise meals for intermittent high intensity exercise [14]. The protocol imitates the activity patterns that occur in soccer matches and includes bouts of walking (31-39s), jogging (41-46s), cruising (41-45s), sprinting (16-21s) and standing stationary. The proportion of each individual activity is similar to those obtained from motion analysis [24] and comprises of $222 \mathrm{~min} 30 \mathrm{~s}$ blocks to comprise one half of $45 \mathrm{~min}$; a total of 4 blocks with a $15 \mathrm{~min}$ half time constituted the whole trial.

During the first $45 \mathrm{~min}$, capillary blood samples were collected and analysed for blood glucose after 10,20 , and $30 \mathrm{~min}$ in order to ascertain if rebound hypoglycaemia was evident in the early stages of exercise. A 15 min half time break was provided during 


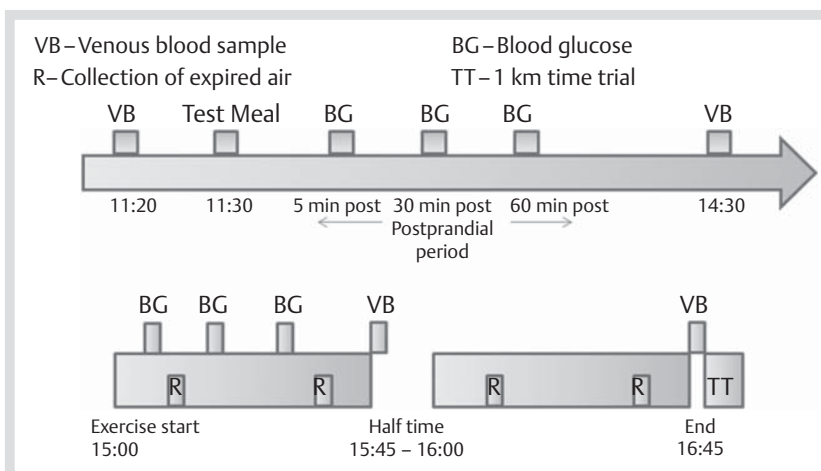

Fig. 1 Schematic of the entire protocol.

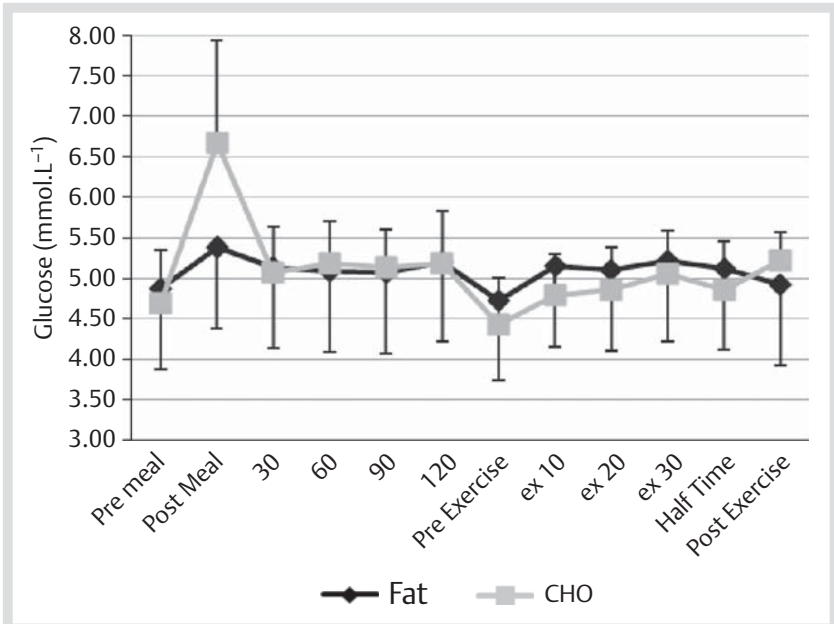

Fig. 2 Blood glucose concentration prior to the test meal, throughout the postprandial period and exercise.

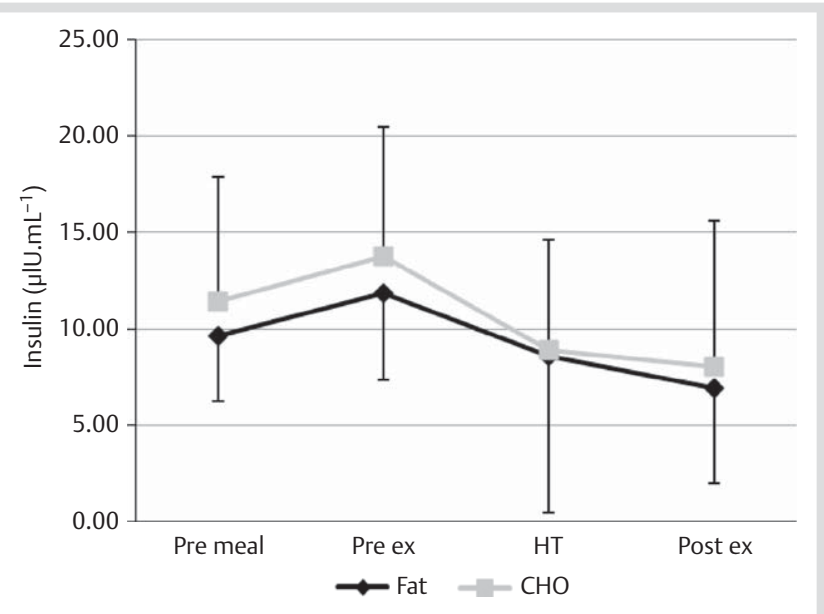

Fig. 3 Insulin concentration prior to the test meal and throughout exercise.

which subjects drank $5 \mathrm{~mL} \cdot \mathrm{kg}^{-1}$ of water and a further venous blood sample was collected. The second $45 \mathrm{~min}$ was a replication of activity patterns experienced during the first $45 \mathrm{~min}$, totalling $90 \mathrm{~min}$ before a final venous blood sample was collected immediately post-exercise and before participants ran the $1 \mathrm{~km}$ time trial. Venous blood samples were collected from the antecubital vein pre-meal, before exercise, at half time, and after exercise, and analysed for fatty acid (FA), glycerol, $\beta$-hydroxybutyrate and lac- tic acid using enzymatic spectrophotometric assays on a RX Daytona clinical chemistry analyser (Randox, Co. Antrim, UK). Serum samples were also analysed for insulin using an Immulite 1000 immunoassay analyser (Siemens Healthcare Diagnostics, Illinois, USA).

At 4 time points during the protocol $(10,35,55$, and $80 \mathrm{~min})$ expired gas was collected for $90 \mathrm{~s}$ using Douglas Bags (Cranlea, Birmingham, UK) and analysed with a Servomex 1440 Gas Analyser (Servomex, Crowborough, UK), from which calculations of fat and $\mathrm{CHO}$ oxidation were made [10].

Subjective measurements of rating of perceived exertion [6] were recorded every $5 \mathrm{~min}$. Heart rate was continually measured and averaged over 5 min periods using a Polar S610 (Polar, Kempele, Finland). A schematic of the protocol can be seen in 0 Fig. 1. The performance test was a $1 \mathrm{~km}$ time trial that was self paced during which participants were blinded to the time and speeds displayed on the treadmill. A performance test rather than a test for exercise capacity (i.e., time to fatigue) was deemed more appropriate and represented a consideration of 'what was left in the fuel tank'. Furthermore, such a high intensity task would necessitate use of all muscle fibres, although the type IIx fibres would be significantly employed [12].

\section{Statistics}

SPSS software (version 17 SPSS, Chicago, IL) was used for data entry and analysis. Analysis of variance for repeated measures on 2 factors (experimental condition and time) was used to analyse differences in the physiologic and metabolic responses in both trials. If a significant interaction was obtained, a least significance difference post hoc test (Bonferroni) was used to determine the location of the variance [5]. A paired t-test was used to analyse time trial performance times. Differences were considered significant at $\mathrm{P}<0.05$. All data are presented as the mean $\pm \mathrm{SD}$.

\section{Results}

$\nabla$

There were no differences between HCM and HFM for blood glucose, insulin and lactate concentrations ( $\bullet$ Fig. $2,3,5$ ), only a significant effect for time $(\mathrm{p}<0.05)$ for blood glucose was apparent. It must be noted that when the area under the insulin-time curve is measured(HCM: $25.6 \pm 13.7 \mu \mathrm{IU} . \mathrm{mL} ; \mathrm{HFM}: 20.9 \pm 11.1 \mu \mathrm{IU}$. $\mathrm{mL})$, although not significant $(\mathrm{p}=0.059)$, it does suggest a tendency that may be of physiological importance.

Significantly higher plasma glycerol and FA concentrations ( $\odot$ Fig. 4) were observed after the HFM than the HCM $(p<0.001)$ and likewise for $\beta$-hydroxybutyrate $(p<0.05)$ ( $\odot$ Fig. 5). All metabolites increased over time $(\mathrm{p}<0.05)$, and significant interactions observed for plasma glycerol and FA $(p<0.005)$. The rate of fat oxidation was also significantly greater after the HFM than the HCM $(p<0.05)$, whereas CHO oxidation was significantly higher after the HCM than the HFM $(p<0.05)$ ( $\bullet$ Fig. 6 ). Fat oxidation was also observed to increase over time $(p<0.05)$ and was approaching significance for an interaction effect $(\mathrm{p}=0.055)$.

Performance completion times were $229.4 \pm 26.5$ s and $228.8 \pm$ $14.4 \mathrm{~s}$ following HCM and HFM respectively, indicating similarities between conditions. Individual analysis observed that 5 participants were faster after HCM and 5 were faster after HFM. In addition, there was no significant effect on performance when data was analysed by test order (Test 1: $226.2 \pm 14 \mathrm{~s}$; Test 2: $231.8 \pm 26.4 \mathrm{~s}$ ). 


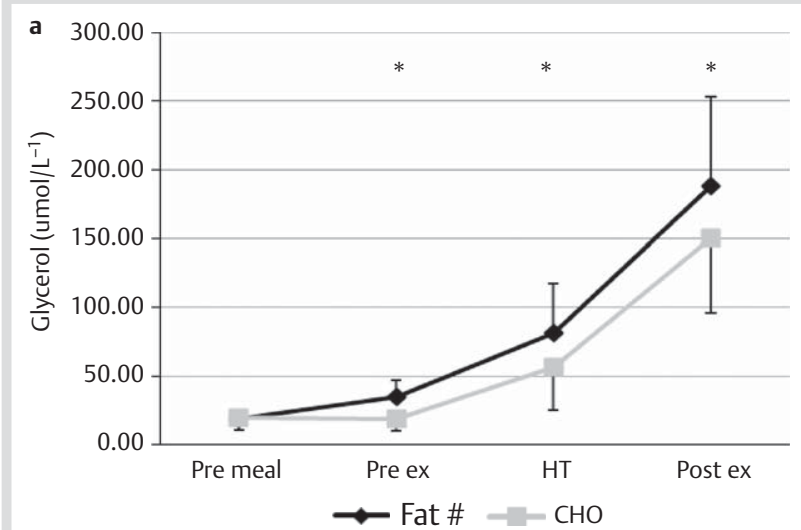

b

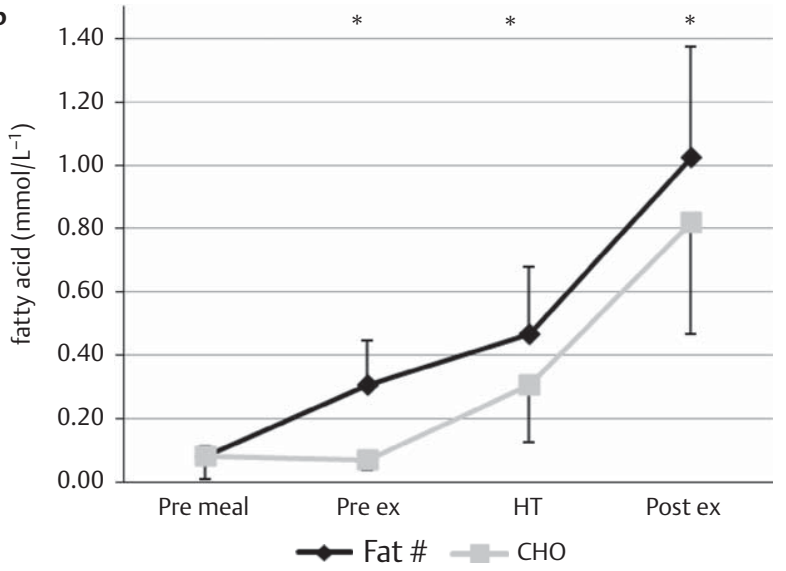

Fig. 4 a Glycerol and b FA concentration prior to the test meal and throughout exercise. \# Indicate a significant main effect. * Indicate where a significant interaction has occurred between time points (Bonferroni corrected paired t-test).

HR and RPE both increased throughout the protocol $(\mathrm{P}<0.001)$, with no difference between conditions (HR-HFM: $145 \pm 5.93, \mathrm{HCM}$ : 146 \pm 7.71 ; RPE - HFM: $13.0 \pm 1.13$, HCM: 13.5 \pm 1.34$)(\mathrm{P}>0.05)$.

\section{Discussion}

$\nabla$

The main finding from this investigation was increased fat oxidation and fat metabolites following the ingestion of a HFM, resulting in similar performance for the 1-km run following the intermittent high intensity exercise. Fat oxidation following consumption of the HFM was $28 \pm 7.71 \%$ greater than that observed following the HCM. Conversely, $\mathrm{CHO}$ oxidation was $35 \pm 6.36 \%$ greater following the HCM compared to the HFM. Previous research [16] observed greater fat oxidation with the fasted control compared to high and low glycaemic meals during a simulated soccer protocol. However, present data indicates an exaggerated increase in fat oxidation with the consumption of a HFM. Furthermore, fat metabolite responses are in accordance with previous research; with significant increases in FA $[21,22,28]$, glycerol $[21,22]$ and $\beta$-hydroxybutyrate [21] after the ingestion of a HFM and during subsequent exercise performance. The observations of FA are consistent with others who report increases over the $90 \mathrm{~min}$ of match play, irrespective of the preexercise nutritional strategies employed [15]. Meals that are high in CHO suppress plasma FA concentrations [28], which may be the case since the HFM contained $59.4 \mathrm{~g}$ of CHO compared to $160 \mathrm{~g}$ with the HCM. Elevated glycerol concentrations following the HFM were
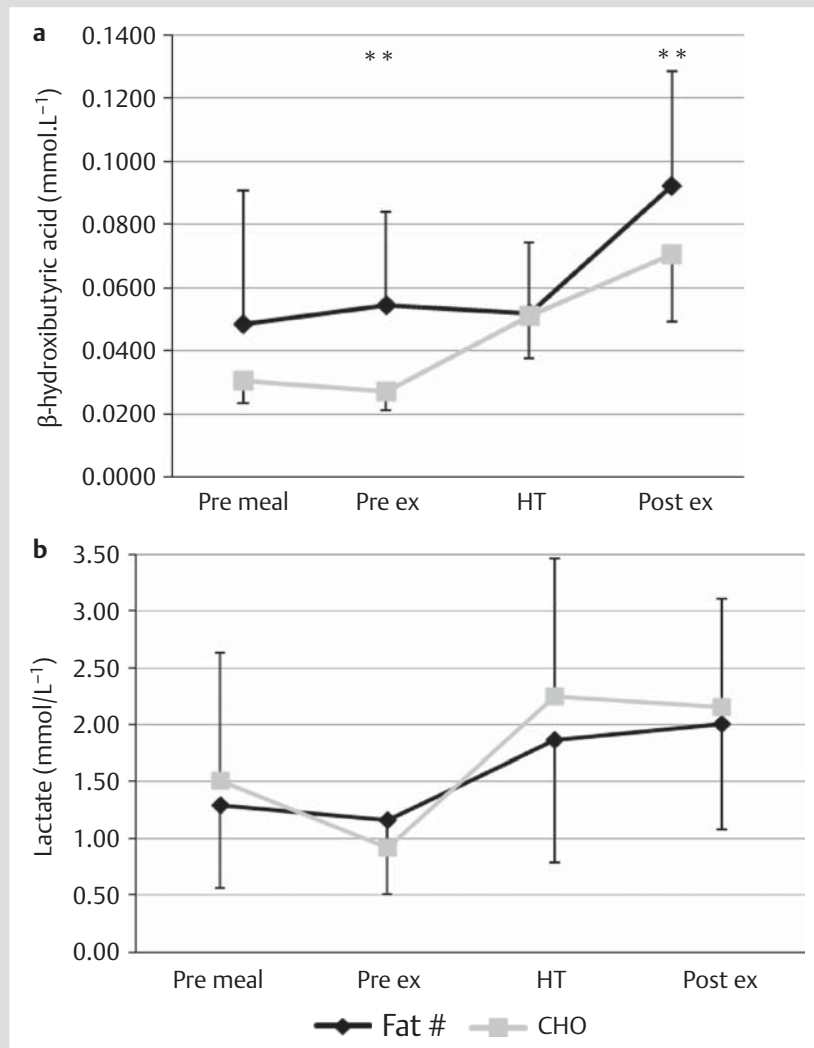

Fig. 5 a $\beta$-hydroxybutyrate and $\mathbf{b}$ Lactate concentration prior to the test meal and throughout exercise. \# Indicate a significant main effect. ** Significant time effect between Pre ex and Post ex.

observed in the current study and are in agreement with others [28]. This finding may be indicative of an enhanced rate of lipolysis. An increase in $\beta$-hydroxybutyrate concentration was also found throughout exercise following ingestion of the HFM, which may reflect stimulation of hepatic ketosis due to greater levels of fat metabolism. This result reflects similar observations in cycling [21]. The presence of elevated concentrations of fat metabolites following the consumption of a HFM relative to the HCM may be attributed to the greater availability of fat following the HFM. However, it may also be a consequence of an elevated insulin concentration preceding the HCM, as insulin is a known antilipolytic hormone that may suppress lipolysis. Notionally, the consumption of a HCM should mediate insulin release and a subsequent inhibition of lipolysis [17]. Whilst no significant effect for insulin concentration was noted between protocols, analysis of the area under the insulin-time curve may suggest a tendency towards a physiological difference favouring the HCM. A limiting factor within the current investigation was the absence of insulin measures during the postprandial period. Montain et al. [19] showed that despite similar postprandial insulin concentrations $4 \mathrm{~h}$ prior to exercise compared to $12 \mathrm{~h}$ postprandially there was a difference in $\mathrm{CHO}$ oxidation and fat metabolites between conditions, supporting a long lasting effect of insulin. Consistent with the current investigation Whitley et al. [28] reported no significant differences in insulin concentrations following the consumption of either a HFM or HCM during exercise. However, again insulin was not analysed postprandially so similar caveats to the present investigations may be warranted. This contrasts the observations from previous research [21] that noted significant elevations in insulin concentrations after a HCM relative to 


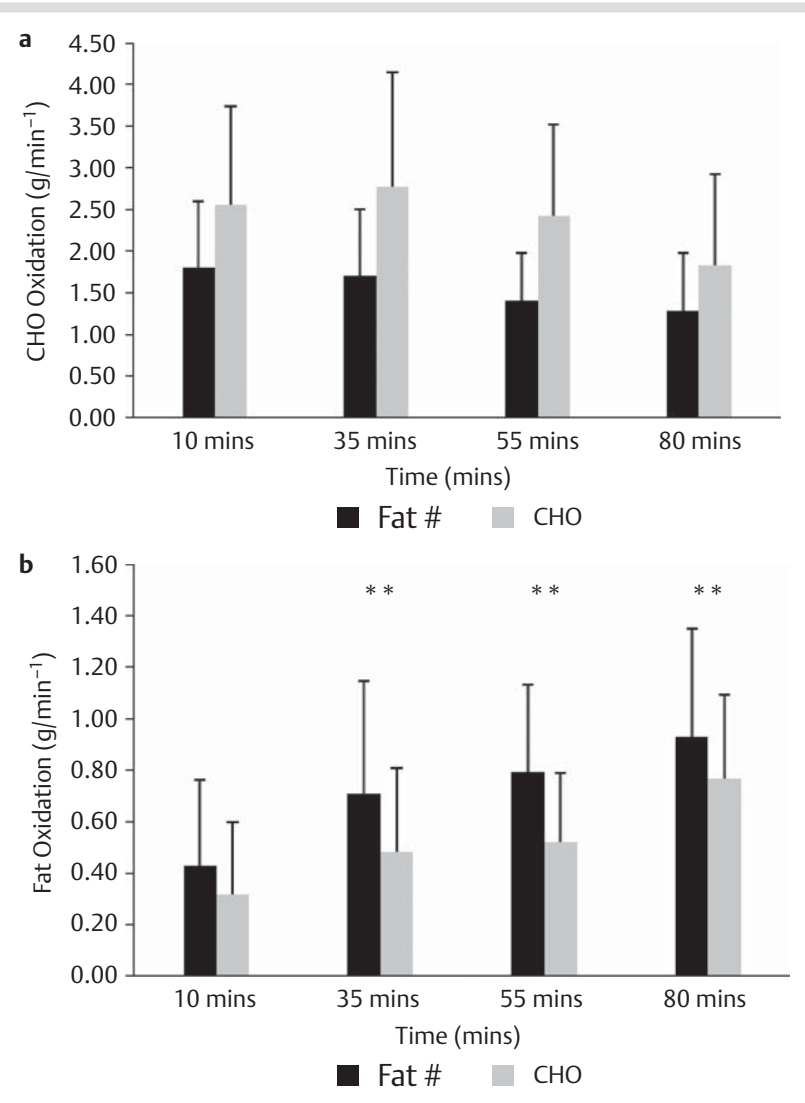

Fig. 6 a $\mathrm{CHO}$ oxidation and $\mathbf{b}$ Fat oxidation measure dudng exercise. \# Indicate a significant main effect. ${ }^{* *}$ Significant time effect between $35 \mathrm{~min}, 55 \mathrm{~min}$ and $80 \mathrm{~min}$.

a HFM. This divergence in insulin responses between the present study and others [21] could be attributed to the volumes and types of $\mathrm{CHO}$ ingested.

Even though significant findings were observed in favour of an increase in fat oxidation and fat metabolites, the current investigation found no difference in the $1 \mathrm{~km}$ time trial performance test following the intermittent high intensity exercise with either test meal (HCM: $229.4 \pm 26.5$ s; HFM: $228.8 \pm 14.4$ s). This performance result was in agreement with previous studies $[21,22,28]$, although other investigations have shown reductions in performance for short term high fat diets that last between 1 day [27], 3 days [4,18,23] or 4 days [11]. A possible explanation for the performance impairments with these studies is the high fat diets they utilise may result in a reduction in muscle and liver glycogen stores prior to exercise. This is unlikely to have occurred in the present study as the participants consumed a normal diet in the $24 \mathrm{~h}$ period leading up to the investigation, which on average consisted of $346.8 \mathrm{~g}$ of $\mathrm{CHO}$, and the same high $\mathrm{CHO}$ breakfast was also consumed on the morning of the test day. The observations that the HFM did not impair performance may be attributable to the additional $\mathrm{CHO}$ intake $(79 \mathrm{~g})$ consumed within the standardised breakfast in addition to the likelihood of an adequate carbohydrate intake in the 24-h prior to the trial ( $350 \mathrm{~g})$.

In conclusion, following a standardised breakfast and an adequate carbohydrate intake over a previous 24 -h period, ingestion of preexercise meals with a high fat or high carbohydrate content produce a similar performance during a $1 \mathrm{~km}$ time trial after a $90-\mathrm{min}$ simulated soccer protocol on the treadmill despite variations in
$\mathrm{CHO}$ and fat oxidation. Under these conditions soccer players may have a broader choice of foods than usually recommended.

\section{References}

1 Agnevik G. Football: Sport Physiology Report Number 7 [in Swedish]. Stockholm: Trygg-Hansa, 1970

2 Balsom PD, Wood K, Olsson P, Ekblom B. Carbohydrate intake and multiple sprint sports: with special reference to football (soccer). Int J Sports Med 1999; 20: 48-52

3 Bangsbo J, Nørregaard L, Thorsøe F. The effect of carbohydrate diet on intermittent exercise performance. Int J Sports Med 1992; 13: 152-157

4 Bergström J, Hermansen L, Hultman E, Saltin B. Diet, muscle glycogen and physical performance. Acta Physiol Scand 1967; 71: 140-150

5 Bland JM, Altman DG. Multiple significance tests: the Bonferroni method. BMJ 1995; 310: 170

6 Borg G. Perceived exertion as an indicator of somatic stress. Scand J Rehabil Med 1970; 2: 93-98

7 Chryssanthopoulos C, Williams C, Nowitz A, Bogdanis G. Skeletal muscle glycogen concentration and metabolic responses following a high glycaemic carbohydrate breakfast. J Sports Sci 2004; 22: 1065-1071

8 Drust B, Reilly T, Cable NT. Physiological responses to laboratory-based soccer-specific intermittent and continuous exercise. J Sports Sci 2000; 18: 885-892

9 Foster-Powell K, Holt SH, Brand-Miller JC. International table of glycemic index and glycemic load values. Am J Clin Nutr 2002; 76: 5-56

10 Frayn $K N$. Calculation of substrate oxidation rates in vivo from gaseous exchanges. J Appl Physiol 1983; 55: 628-634

11 Galbo H, Holst IJ, Christensen NJ. The effect of different diets and of insulin on the hormonal response to prolonged exercise. Acta Physiol Scand 1979; 10: 19-32

12 Greenhaff PL, Nevill ME, Soderlund K, Bodin K, Boobis LH, Williams C, Hultman $E$. The metabolic responses of human type I and II muscle fibres during maximal treadmill sprinting. J Physiol 1994; 1: 149-155

13 Harriss DJ, Atkinson G. Update - ethical standards in sport and exercise science research. Int J Sports Med 2011; 32: 819-821

14 Hulton AT, Gregson W, MacLaren DPM, Doran DP. Effects of GI meals on intermittent exercise. Int J Sports Med 2012; 33: 459-462

15 Krustrup P, Mohr M, Steensberg A, Bencke J, Kiaer M, Bangsbo J. Muscle and blood metabolites during a soccer game: implications for sprint performance. Med Sci Sports Exerc 2006; 38: 1-10

16 Little JP, Chilibeck PD, Ciona D, Vandenberg A, Zello GA. The effects of low- and high-glycemic index foods on high intermittent exercise. Int J Sport Physiol Perform 2009; 4: 367-380

17 Little JP, Chilibeck PD, Ciona D, Forbes S, Rees H, Vandenberg A, Zello $G A$. Effect of low- and high-glycemic-index meals on metabolism and performance during high intermittent exercise. Int J Sports Nutr Exerc Metab 2010; 20: 447-456

18 Martin B, Robinson S, Robertshaw D. Influence of diet on leg uptake of glucose during heavy exercise. Am J Clin Nutr 1978; 31: 62-67

19 Montain SJ, Hopper MK, Coggan AR, Coyle EF. Exercise metabolism at different time intervals after a meal. J Appl Physiol 1991; 70: 882-888

20 Neufer PD, Costill DL, Flynn MG, Kirwan JP, Mitchell JB, Houmard J. Improvements in exercise performance: effects of carbohydrate feedings and diet. J Appl Physiol 1987; 62: 983-988

21 Okano G, Sato Y, Takumi Y, Sugawara M. Effect of $4 \mathrm{~h}$ preexercise high carbohydrate and high fat meal ingestion on endurance performance and metabolism. Int J Sports Med 1996; 17: 530-534

22 Okano G, Sato Y, Murata Y. Effect of elevated blood FFA levels on endurance performance after a single fat meal ingestion. Med Sci Sports Exerc 1998; 30: 763-768

23 Pitsiladis YP, Maughan RJ. The effects of exercise and diet manipulation on the capacity to perform prolonged exercise in the heat and in the cold in trained humans. J Physiol 1999; 517: 919-930

24 Reilly T, Thomas $V$. A motion analysis of work-rate in different positional roles in professional soccer match play. J Hum Mov Stud 1976; 7: 87-97

25 Saltin B. Metabolic fundamentals in exercise. Med Sci Sports Exerc 1973; 5: 137-146

26 Sherman WM, Brodowicz G, Wright DA, Allen WK, Simonsen J, Dernbach A Effects of $4 \mathrm{~h}$ preexercise carbohydrate feedings on cycling performance. Med Sci Sports Exerc 1989; 21: 598-604

27 Starling RD, Trappe TA, Parcell A, Kerr CG, Fink WJ, Costill DL. Effects of diet on muscle triglyceride and endurance performance. J Appl Physiol 1997; 82: 1185-1189

28 Whitley HA, Humphreys SM, Campbell IT, Keegan MA, Jayanetti TD, Sperry DA, MacLaren DP, Reilly T, Frayn KN. Metabolic and performance responses during endurance exercise after high-fat and highcarbohydrate meals. J Appl Physiol 1998; 85: 418-424 